\title{
Séance du Comité central du 17 décembre 2010
}

Recherche sur les soins - La FMH participera aux côtés d'autres partenaires du corps médical au financement du Programme de recherche sur les soins de l'Institut de médecine sociale et préventive de l'Université de Berne.

\section{Révision de la structure tarifaire «TARVISION»}

- Conformément à la décision du Comité central (CC) et de l'Assemblée des délégués de la FMH, les sociétés de discipline ont commencé les travaux de révision de la structure tarifaire TARMED. Elles ont été chargées:

- d'adapter la structure tarifaire avec minutages, valeur intrinsèque et unités fonctionnelles, indépendamment des coûts;

- d'adapter le modèle de coûts (loyers, salaires, etc.) aux conditions actuelles

- d'élaborer un chapitre consacré uniquement aux médecins de premier recours;

- et de mettre en évidence enfin les coûts réels.

Une fois ces travaux terminés, une solide base de données viendra étayer notre argumentation lors des négociations tarifaires avec les assureurs.
Alliance DRG-Personnel - Le CC a pris position sur les demandes de l'Alliance DRG-Personnel dans le cadre de l'introduction de SwissDRG. Selon la FMH, l'accord visé doit être converti en une déclaration d'intention, les signataires ne pouvant, pour des raisons juridiques, mettre en œuvre eux-mêmes les points énoncés. La FMH cosignera cette déclaration d'intention.

Fondation pour la Sécurité des Patients - Le CC de la FMH soutient le projet «Climat de sécurité dans les cabinets de médecine de premier recours en Suisse».

\section{Stratégie d'élimination de la rougeole - La} FMH approuve la stratégie d'élimination de la rougeole. Celle-ci a notamment pour objet les points suivants:

- rémunération convenable de la prestation pour le conseil, la promotion et la vaccination;

- accès facilité au vaccin;

- aucune mesure de contrainte.

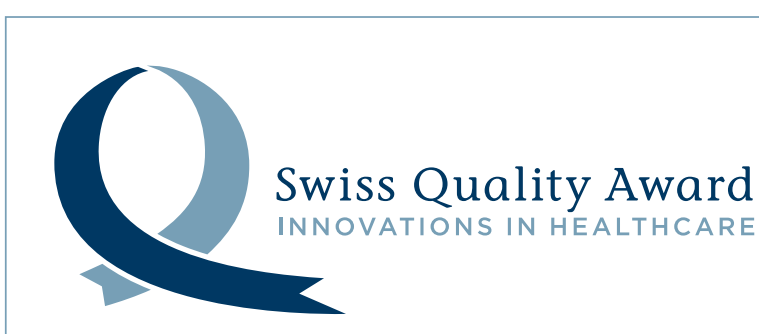

\section{ENVOYEZ VOS PROJETS AVANT LE 15 MARS 2011}

Le Swiss Quality Award récompense les meilleures innovations en matière de qualité dans le système de santé. Participez et enregistrez votre projet sur le site www.swissqualityaward.ch. 